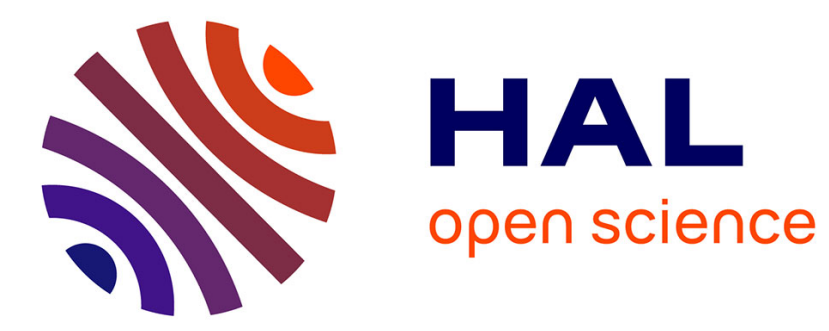

\title{
Computer Vision Based Nacre Thickness Measurement of Tahitian Pearls
}

Martin Loesdau, Sébastien Chabrier, Alban Gabillon

\section{To cite this version:}

Martin Loesdau, Sébastien Chabrier, Alban Gabillon. Computer Vision Based Nacre Thickness Measurement of Tahitian Pearls. The International Conference on Quality Control by Artificial Vision 2017, May 2017, Tokyo, Japan. pp.103381C, 10.1117/12.2266924 . hal-03132310

\section{HAL Id: hal-03132310 https://hal-upf.archives-ouvertes.fr/hal-03132310}

Submitted on 5 Feb 2021

HAL is a multi-disciplinary open access archive for the deposit and dissemination of scientific research documents, whether they are published or not. The documents may come from teaching and research institutions in France or abroad, or from public or private research centers.
L'archive ouverte pluridisciplinaire HAL, est destinée au dépôt et à la diffusion de documents scientifiques de niveau recherche, publiés ou non, émanant des établissements d'enseignement et de recherche français ou étrangers, des laboratoires publics ou privés. 


\title{
Computer Vision Based Nacre Thickness Measurement of Tahitian Pearls
}

\author{
Martin Loesdau, Sébastien Chabrier, Alban Gabillon \\ Laboratoire d'Excellence CORAIL GePaSud EA 4238, Université de la Polynésie française \\ Punaauia, Polynésie française
}

\begin{abstract}
The Tahitian Pearl is the most valuable export product of French Polynesia contributing with over 61 million Euros to more than $50 \%$ of the total export income. To maintain its excellent reputation on the international market, an obligatory quality control for every pearl deemed for exportation has been established by the local government. One of the controlled quality parameters is the pearls nacre thickness. The evaluation is currently done manually by experts that are visually analyzing X-ray images of the pearls. In this article, a computer vision based approach to automate this procedure is presented. Even though computer vision based approaches for pearl nacre thickness measurement exist in the literature, the very specific features of the Tahitian pearl, namely the large shape variety and the occurrence of cavities, have so far not been considered. The presented work closes the. Our method consists of segmenting the pearl from X-ray images with a model-based approach, segmenting the pearls nucleus with an own developed heuristic circle detection and segmenting possible cavities with region growing. Out of the obtained boundaries, the 2-dimensional nacre thickness profile can be calculated. A certainty measurement to consider imaging and segmentation imprecisions is included in the procedure. The proposed algorithms are tested on 298 manually evaluated Tahitian pearls, showing that it is generally possible to automatically evaluate the nacre thickness of Tahitian pearls with computer vision. Furthermore the results show that the automatic measurement is more precise and faster than the manual one.
\end{abstract}

Keywords: pearl quality control, X-ray imaging, computer vision, X-ray image segmentation, Tahitian pearls

\section{INTRODUCTION}

The Tahitian pearl is a precious gem that is cultivated in the clear warm lagoons of French Polynesia. Due to its mystical dark colors and its brilliant luster the 'Queen of Pearls' achieves high prices on the international market. Accordingly, it is a vital source of income for the French Polynesian territory. In 2015 loose Tahitian pearls amounting to over 61 million Euros were exported, being with over $50 \%$ by far the most significant source of export income. According to resolution $n^{0} 2005-42$ issued by the French Polynesian government on February $4^{\text {th }}$ in 2005 , all Tahitian pearls that are exported to foreign countries must have been officially validated as being of sufficient quality. The governmental organization currently in charge of this obligatory control is the Direction des Ressources Marines et Minières (DRMM, administration for marine and mineral resources). One of the crucial quality parameters defined in the resolution is the pearls nacre thickness. It is written that the minimal nacre thickness has to be at least $0.8 \mathrm{~mm}$, otherwise the pearl cannot be exported. Currently, the obligatory quality control is done manually by 10 experts for over 7 million pearls that are supposed to be exported annually. As the amount of pearls already exceeds the control capacity of the DRMM, the nacre thickness is not evaluated for each pearl specifically, albeit demanded by the mentioned resolution. Usually a random sample is taken out of a set of pearls from one pearl farmer and if several pearls do not pass the control the rest of the set is controlled as well. Otherwise, the whole set is expected to be generally of sufficient quality. Still, the control procedure has repeatedly been criticized by pearl exporters as being slow and partially imprecise. A latency of several weeks between the disposal of pearls at the DRMM and the control result is the rule rather than an exception. As for the manual evaluation of the nacre thickness X-ray images are taken anyway, an automation of this process with image processing techniques suggests itself. The results of our work on automatizing the nacre thickness measurement of Tahitian pearls with computer vision applied to X-ray images of the pearls is presented in this article.

Even though the general goal of our project is to fully automatize the nacre thickness evaluation of Tahitian pearls, this stage of the project is focused on X-ray image processing techniques. An automatized X-ray image acquisition and the routing of pearls into and out of the X-ray machine will be done in a future work. 
Our method to automatically measure the nacre thickness of Tahitian pearls consists of five sub steps that will be introduced in section 3: (1) segmenting the pearl from the original X-ray images, (2) detecting the nucleus with in the pearl, (3) detecting cavities, (4) calculating the 2D nacre thickness profile and (5) identifying pearls with $20 \%$ of its nacre thickness lower than $8 \mathrm{~mm}$ according to the manual procedure currently done at the DRMM. The proposed algorithms were tested with a set of 298 pearls that were classified manually by experts of the DRMM. The results are presented and discussed in section 4 , showing that it is generally possible to automatically measure the nacre thickness of Tahitian pearls. Furthermore the results show that the automatic measurement is more precise than the manual one. A conclusion with remarks on future work is given in section 5.

\section{RELATED WORK}

For the purpose of analyzing visually the internal structure of pearls, different X-ray based methods have been deemed suitable, such as X-ray microtomography ([1] and [2]) or X-ray phase contrast and scattering [3]. As an alternative, optical coherence tomography (OCT) is proposed in [4]. An integrated system that captures OCT images and spectral data based on fluorescence spectroscopy is described in [5], allowing an analysis of both, internal structure as well as for example color properties.

While the mentioned articles focus on a suitability analysis of the used imaging methods by visual data interpretation, research on the mechanical aspects of computer vision based pearl quality assessment can be found in [6]. Here, a computer vision system is introduced, including remarks on mechanical components, image capturing and algorithmic processing to grade the pearls shape and size. Alternative solutions for the proposed pearl routing in pipes can be found in [7], in which pearls are moved with a mechanical arm that picks pearls separately by suction.

Strongly related to our work is the article [8], in which an automated nacre thickness measurement of pearls is proposed. The pearl type is not specified, but the origin of the article suggests that Chinese Freshwater pearls are used. For image acquisition, optical coherence tomography (OCT) is applied. The obtained OCT images are denoised and edge pixels of the outer boundary of the pearl and the nucleus are identified with Canny edge detection and Support Vector Machine respectively. Afterwards the outer boundary of the pearl and the nucleus are obtained by fitting circles to the extracted edge pixels. The automatic nacre thickness measurement is evaluated with 8 manually segmented pearls and reaches 93.6\% average accuracy. While an interesting and straight-forward approach, it is stated clearly in the article that the basic assumption for this approach is that the outer boundary of a pearl can be described by a circle: 'As a priori knowledge, we suppose that the pearl and nucleus are both spheres, thus, in 2D OCT image, the outer and inner boundaries of nacre are both circles'. This assumption does not hold for Tahitian pearls, as shape variety is a typical feature. In Figure 1 on the left, an image of the cited article shows the fitted circles in blue that describe the outer boundary of the pearl and the nucleus. The X-ray images of two typical Tahitian pearls on the right show that their outer boundary cannot be described with circles. The approach is further developed in [9] and [10], but still under the condition that the contours of pearl and nucleus can be approximated by circles. Additionally, the occurrence of cavities is not addressed, as they are not typical for Chinese Freshwater Pearls. The approach is hence not applicable to Tahitian pearls.

Compared to our previously published approach [11], an acceleration of the measurement of 50\% could be reached by replacing the relatively time-intense active contour approaches by a model-based approach for the pearl segmentation and a region growing approach for cavity detection. An additional advantage of the model-based pearl segmentation is that only information concerning the background is used, meaning the approach is independent of the varying pearl geometry. Furthermore, a certainty measurement was added to the evaluation procedure and the test image database was significantly increased to 298 samples.

Lei et al.

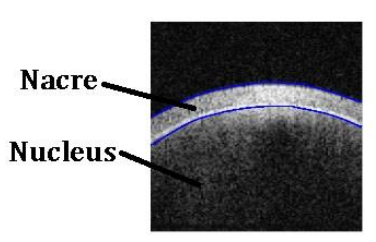

Tahitian Pearls

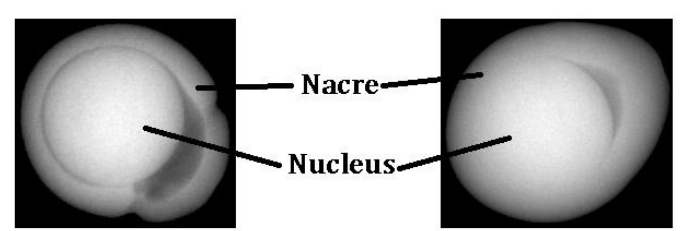

Figure 1: Results of the approach proposed in [8] based on the assumption that the pearl boundary can be described by a circle (left). On the right two typical examples of Tahitian pearls are shown for which this assumption does not hold. 


\section{AUTOMATIC NACRE THICKNESS MEASURMENT}

Analyzing X-ray images of pearls (visually or numerically) means generally to differentiate between three characteristic regions. The regions are visualized in Figure 2 with a mechanically bisected pearl (second image, taken from [2]) and an X-ray image of a similar pearl (third image). Within the pearl is the nucleus (a blank nucleus is shown in the image on the left), an artificially, out of the shell from a freshwater mollusk formed sphere (marked with number 1). In the X-ray image, the nucleus appears as a circular object within the pearl. The black region of the bisected pearl is the nacre that was secreted by the pearl oyster around the nucleus (marked with number 2). In several cases the nacre does not connect directly to the nucleus. Between nucleus and nacre is accordingly a cavity (marked with number 3 ). Cavities appear in the X-ray images as darker regions within the pearl.

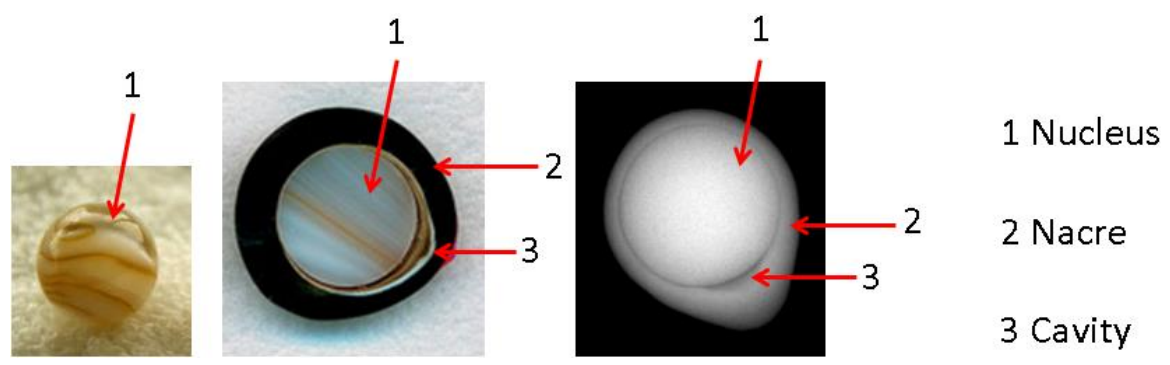

Figure 2: Blank nucleus (left), bisected Tahitian pearl (middle, taken from [2]) and X-ray image of a Tahitian pearl that is similar to the bisected one (right).

The possibility to visually evaluate the nacre thickness depends on if and how much cavities separate the nacre from the nucleus. Figure 3 shows some typical cases. In the first column, two pearls with a cavity circumferential to the nucleus that are easily evaluable visually can be seen. The second column shows two pearls with a slight cavity around the nucleus. While the pearls of the first column can be evaluated in a glimpse, these cases are still evaluable but need already a closer look. The third column shows two pearls with a non-circumferential cavity. Even though the nucleus is only partially visible, the general contour of the nucleus can be estimated cognitively. The fourth column shows two pearls without any cavity. As their nacre thickness cannot be estimated visually, those pearls pass the current quality control and can be exported.

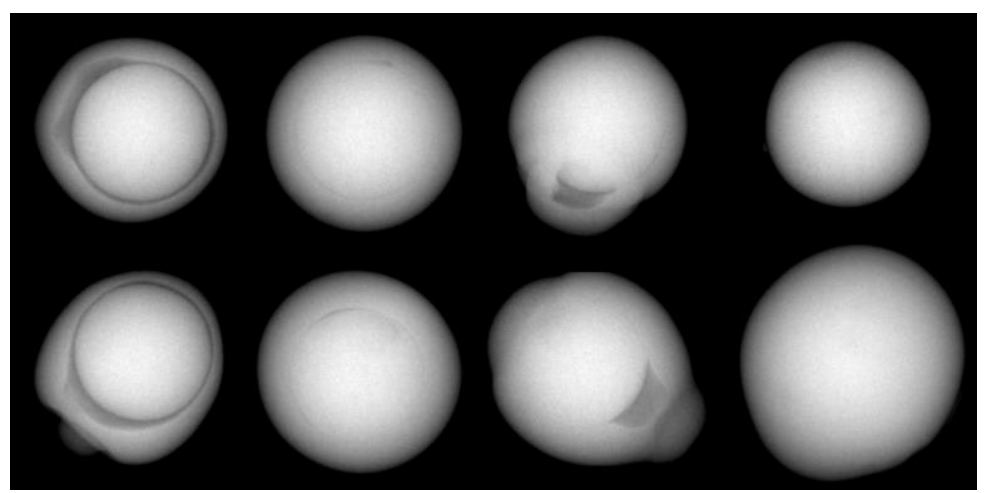

Figure 3: Different nacre configurations of Tahitian pearls.

\subsection{Image Acquisition and Processing}

For the acquisition of the X-ray images an X-TEK XT V 160 PCB inspection system was used. The parameter configuration used by the employees of the DRMM was kept (voltage: $130 \mathrm{k}$, current $35 \mu A$, averaging: 8 images per output image). All obtained images have a pixel resolution of $1000 \times 1000$ as a fixed size determined by the X-ray detection device of the X-ray machine. All images are 16 bit greylevel images of uncompressed TIFF format. All algorithms were programmed and run in Matlab 7.12.0 (R2012a). All tests were run under Windows 764 bit on a dualcore Samsung R590 (CPU Intel Core i5 450M / 2.4 GHz) with 4GB RAM. 


\subsection{Image Configuration}

Images were taken similar to the manual procedure of the DRMM. A support with boreholes was filled with pearls and placed into the X-ray machine (Figure 4 on the left shows a detail of the support). Afterwards, images were taken pearl by pearl. The resulting images contain the gradient of the borehole and one pearl each (Figure 4, two example images on the right).
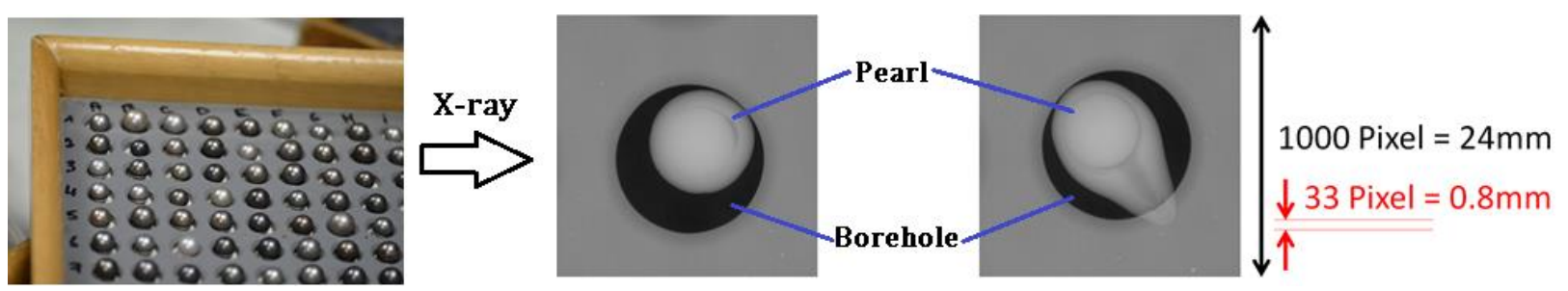

Figure 4: Pearls within the support (left) and two examples of the obtained test image configuration.

\subsection{Pearl Segmentation}

To segment the pearl, a model based approach was developed that is based on the geometry of the used support and the physical properties of X-ray attenuation. A synthetic background image is generated according to the current image configuration and both (original and synthetic) are calibrated so that their intensity corresponds to the geometry of the radiographed scene (Figure 5 first two columns). Due to this calibration, the synthetic image can be mathematically subtracted from the original image, which corresponds to an X-ray image of a pure pearl taken without the support (Figure 5 top right image). Resulting artefacts are cleaned with a smoothing filter operation and the pearl identified by thresholding (Figure 5 bottom right image).

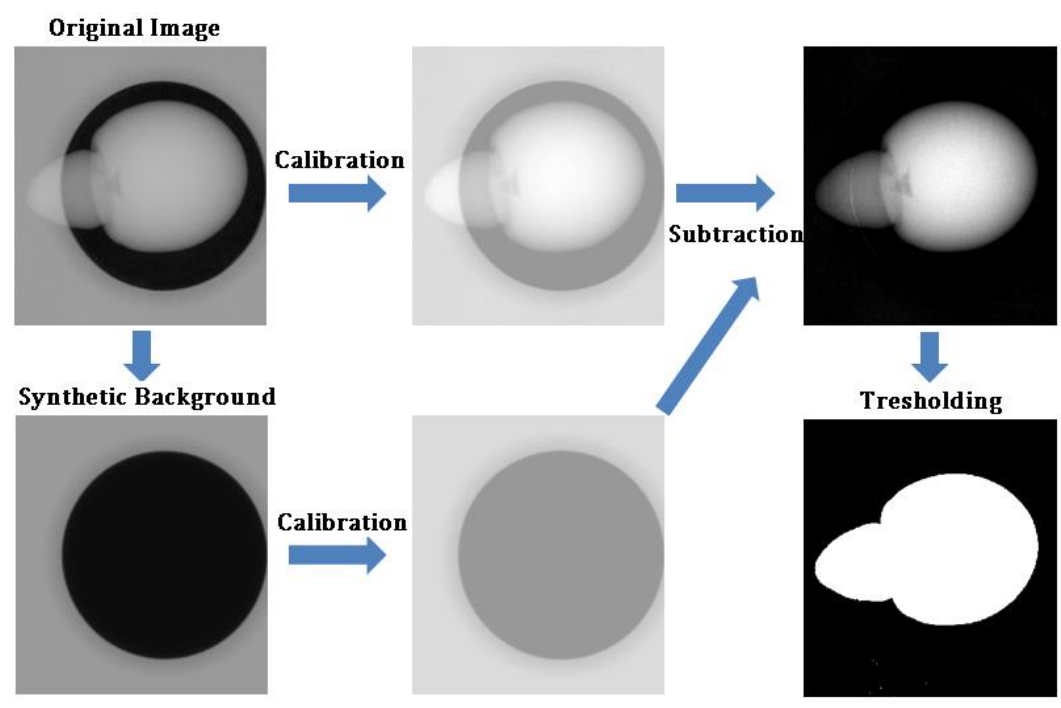

Figure 5: Workflow of the segmentation of the pearl.

\subsection{Nucleus Detection}

As the size of the nucleus is not known prior to the measurement, its radius and center have both to be detected. For this purpose a new heuristic circle detection algorithm was developed (see as well [11]). A small circle is initialized around the region of highest image intensity, as after the prior calibration and segmentation it is known to belong to the nucleus (first image in Figure 6). A logical boundary probability is formulated that pushes the circle away from probable nucleus boundary pixels, while its radius is iteratively increased (the blue vectors in Figure 6 are circle pixels with boundary probability 1 , the gaps between the vectors are circle pixels with boundary probability 0 ). If the circle is successfully pushed away from the nucleus boundary at each iteration, it will at one point cover the boundary of the nucleus (third image in Figure 6). 


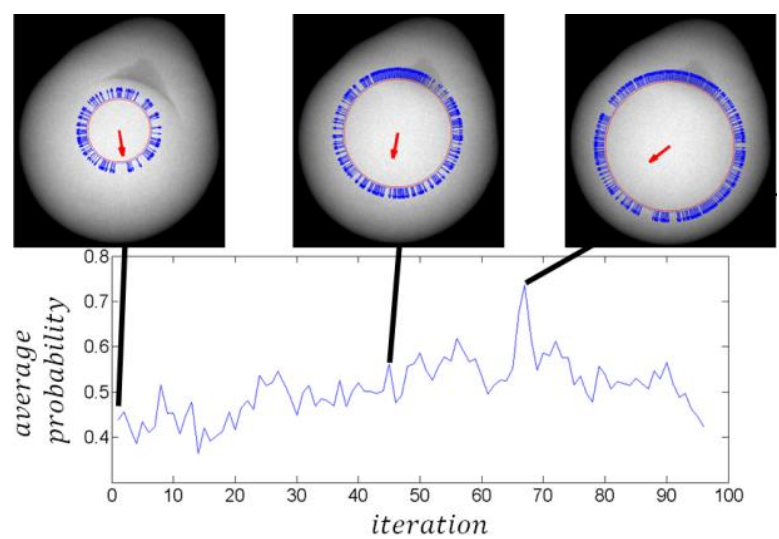

Figure 6: Schema of the developed iterative approach to detect the nucleus.

\subsection{Cavity Detection}

To detect possible cavities, a classical region growing procedure is applied. Seed pixels are the previously obtained outer boundary of the pearl, as it is known to be nacre (second image of Figure 7). According to a simplified physical model, cavity boundaries are defined by local intensity maxima. The region growing is accordingly stopped at local maxima or if the previously detected nucleus is reached (last image of Figure 7).
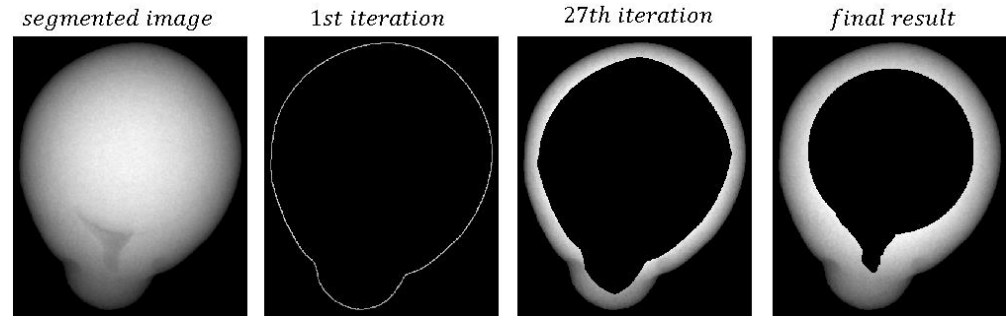

Figure 7: Region growing procedure to detect cavity boundaries.

\subsection{Nacre Thickness Profile}

After the three segmentation steps, all crucial boundaries for the calculation of the nacre thickness profile are determined (Figure 8 left two images). The nacre thickness is measured for each pixel of the obtained outer boundary of the pearl in direction of its inside pointing normal vector (Figure 8 third image). This procedure corresponds to the current manual nacre thickness evaluation at the DRMM. The result is a vector containing the distance in pixel to either the nacre or the nucleus for each boundary pixel. Together with the spatial resolution of the images the nacre thickness profile can be expressed in $\mathrm{mm}$ (Figure 8 on the right).
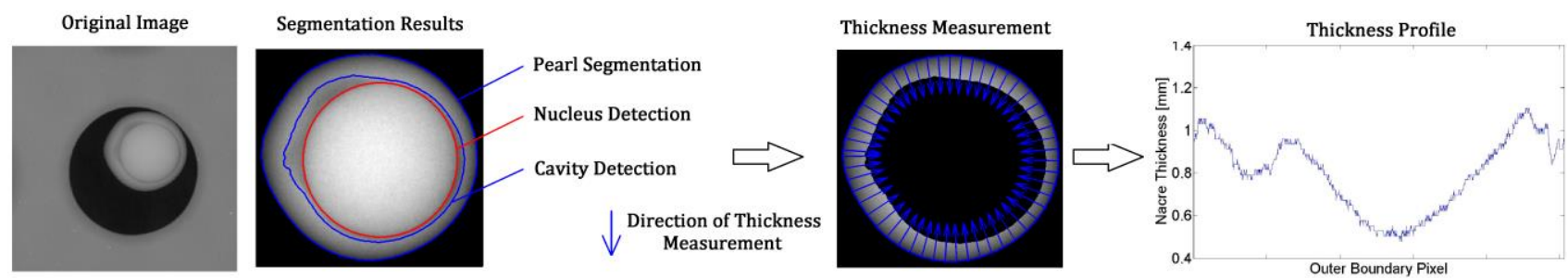

Figure 8: Obtained boundaries after applying the three segmentation steps (left) and schematic representation of the nacre thickness measurement in direction of the inside pointing normal vectors of the detected outer boundary (blue arrows in the third image). The graph on the right is the obtained nacre thickness profile in $\mathrm{mm}$. 


\subsection{Identification of Rejects and Certainty Value}

The procedure currently applied at the DRMM to decide whether to reject a pearl from exportation or not is to identify visually if more than $20 \%$ of the whole nacre region has a thickness lower than $0.8 \mathrm{~mm}$. The implementation is accordingly. The amount of boundary pixels with a nacre thickness lower than $0.8 \mathrm{~mm}$ is divided by the sum of boundary pixels. This procedure is visualized in Figure 9. The graph on the left shows the calculated nacre thickness for every boundary pixels in $\mathrm{mm}$. The dashed line is the current threshold of $0.8 \mathrm{~mm}$. Every entry of the nacre thickness profile vector lower than $0.8 \mathrm{~mm}$ is marked in red, as well as the corresponding regions in the X-ray image of the pearl on the right. The percentage of the nacre region lower than $0.8 \mathrm{~mm}$ is in the given example $46 \%$ which makes the pearl a reject in the current definition of the DRMM.
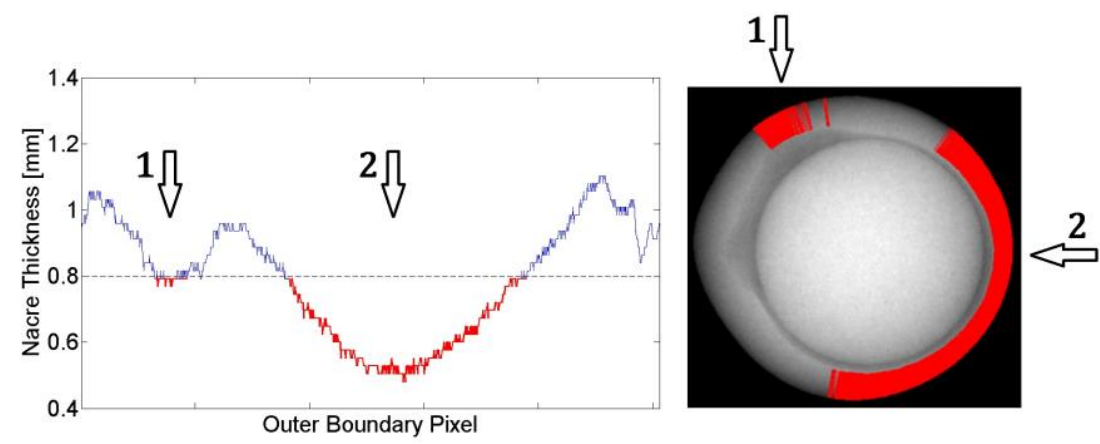

Figure 9: Automatically measured nacre thickness profile of a Tahitian pearl (left). The regions of the profile with a thickness lower than $0.8 \mathrm{~mm}$ and the corresponding regions of the X-rayed pearl are marked in red

As the automated measurement as well as the general imaging of spherical objects result in a discrepancy between detected boundaries and real boundaries, a certainty measurement for the obtained results is proposed. The detected outer boundary is pixel wise shifted along its outside pointing normal vectors which results in a larger nacre thickness measurement. At each shift, the percentage of the current nacre thickness profile lower than $0.8 \mathrm{~mm}$ is measured (Figure 10 on the right, the ordinate describes the shift in pixels and the abscissa the percentage of nacre thickness lower than $0.8 \mathrm{~mm}$ at the corresponding shift.). In the example of Figure 10, less than $20 \%$ of the nacre thickness is lower than $0.8 \mathrm{~mm}$ when the outer boundary is shifted by 9 pixels. It means the automatic detection of the example pearl as a reject would be false if imprecisions of all segmentation steps and imaging imprecisions resulted in a lower nacre thickness of 9 pixels at every point of the outer boundary.

As the average precision of each segmentation step lies at $\sim$ pixel, pearls automatically detected as rejects with a certainty value lower than 5 pixels might still be considered as good for exportation, according to the paradigm that in case of doubt, pearls should not be rejected. However, the certainty value should be seen as a variable that is to be adjusted by the DRMM according to the results of the test phase of the prototype of our algorithm.
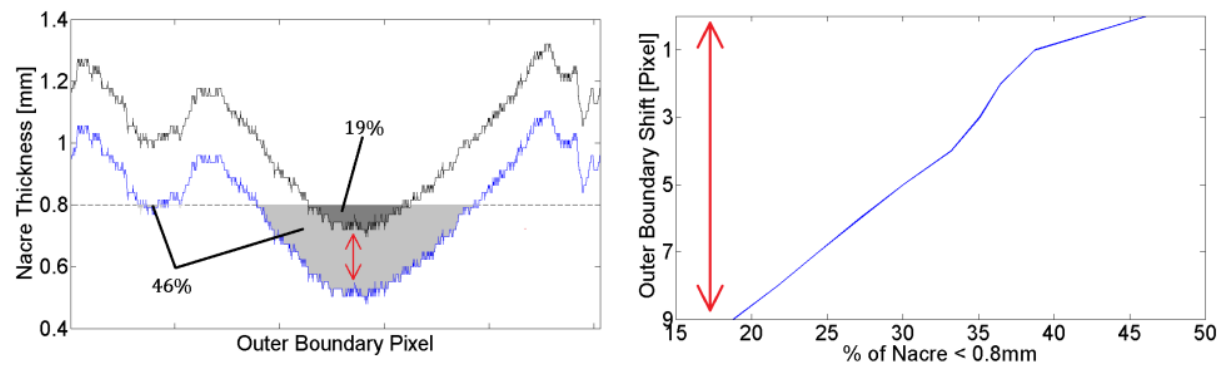

Figure 10: Representation of the certainty calculation. The graph on the left shows the automatically calculated nacre thickness profile in $\mathrm{mm}$ blue, and the by 9 pixels shifted profile in black. The graph on the right shows the percentage of nacre thickness lower than $0.8 \mathrm{~mm}$ at each boundary shift from 0 to 9 pixels. 


\section{TEST RESULTS AND DISCUSSION}

For evaluation purpose, X-ray images of 298 Tahitian pearls were acquired at the DRMM. The pearls were classified manually by experts of the DRMM according to their daily routine in rejects or good for exportation. Afterwards, all images were processed as explained in the previous section. The graph in Figure 11 shows the certainty values of all pearls that were automatically classified as rejects. All pearls that were manually classified as rejects were as well automatically classified as to reject (red bars in Figure 11). Additionally, 26 pearls were automatically classified as to reject but classified manually as to be good for exportation (blue bars). A visual analysis of the segmentation results of these pearls by DRMM experts showed that all obtained boundaries are correctly assessed (6 examples are shown in Figure 12). Anyhow, as mentioned earlier, pearls that are automatically detected as to reject with a certainty value lower than $\sim 5$ pixels might be considered as to be good for exportation, as, due to the nature of the images as well as their processing, an absolute precision cannot be guaranteed.

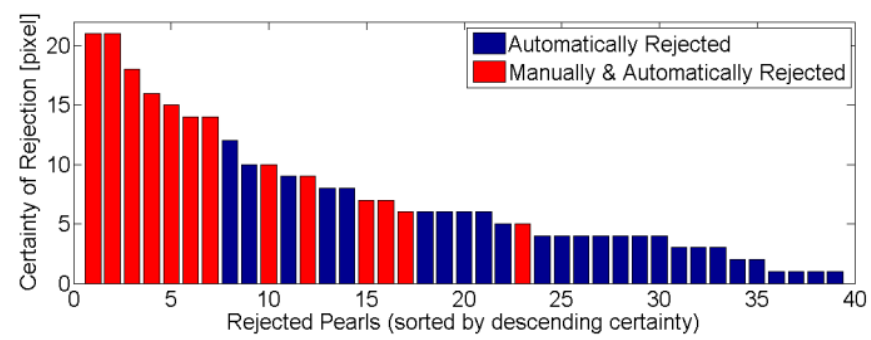

Figure 11: Rejection certainty values of the automated detection of pearls with $20 \%$ nacre thickness lower than $0.8 \mathrm{~mm}$. Red bars correspond to pearls manually classified as rejects by DRMM experts.

The results generally show that, first of all, it is generally possible to automatically evaluate the nacre thickness of Tahitian pearls out of X-ray images, despite the large variation of shape and nacre-cavity-nucleus configuration. Secondly, the automatic classification is more reliable as the manual one. This is due to the fact that the experts do not always have enough time to analyze region boundaries in detail, seeing that thousands of pearls have to be controlled on a daily basis. According to the paradigm that in case of doubt the decision has to be made in favor of pearl quality, pearls with a thickness close to $0.8 \mathrm{~mm}$ might slip through the control. Another challenge of the manual classification is the estimation if a region that has a thickness lower than $0.8 \mathrm{~mm}$ corresponds to $20 \%$ of the $2 \mathrm{D}$ nacre profile. This is specifically for non-round pearls a non-trivial task. Here, the automatic detection has a clear advantage, as the percentage in regards of the complete outer boundary can be calculated precisely. The average processing time for the complete nacre thickness measurement with the specified equipment is 0.8 seconds per image. As the manual evaluation of a pearl lies, depending on the complexity of the nacre configuration in the range of 1 to 2 seconds, an acceleration of the quality control is hence feasible with computer vision.
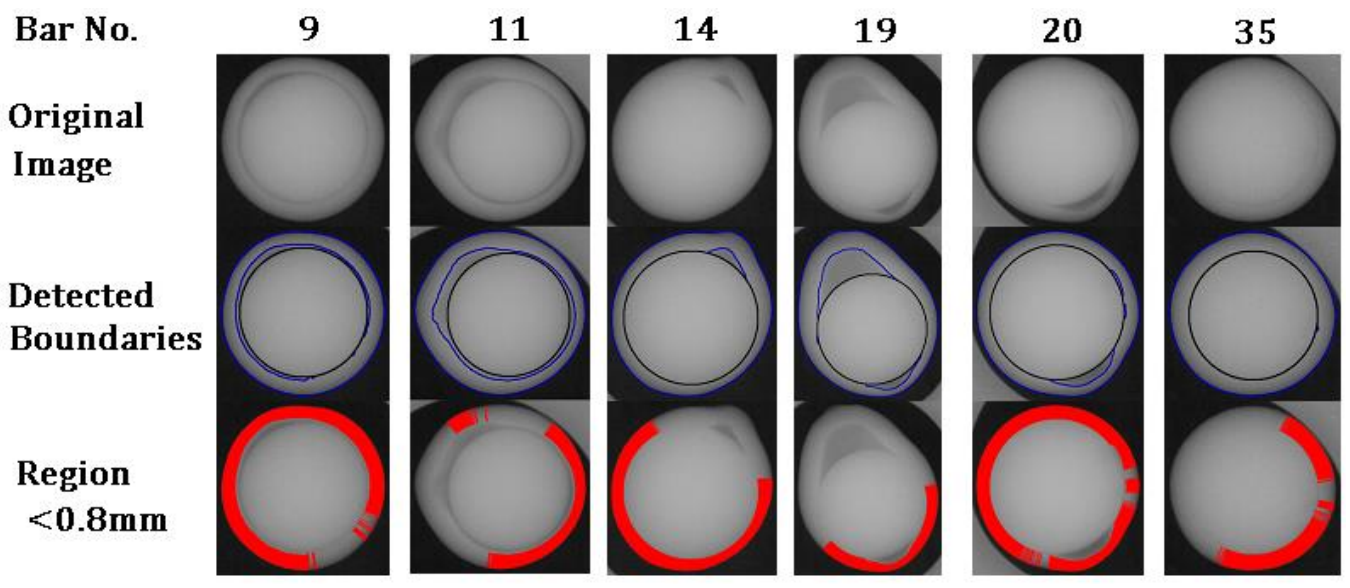

Figure 12: Six examples of pearls that were manually classified as good but automatically classified as rejects. The bar number on top of each row corresponds to the bar plot in Figure 11. 


\section{CONCLUSION AND FUTURE WORK}

In the previous sections our approach to automatically measure the nacre thickness of Tahitian pearls out of X-ray images has been presented. The goal of this work is to automate the obligatory quality control of Tahitian pearls deemed for exportation, a procedure that is currently done manually. The proposed algorithms were tested on 298 manually classified Tahitian pearls showing that a computer vision based nacre thickness measurement of Tahitian pearls is faster and more reliable than the human visual classification. Even though computer vision based approaches for pearl nacre thickness measurement exist in the literature, the very specific features of the Tahitian pearl, namely the large shape variety and the occurrence of cavities, have so far not been considered, making the presented approach a novelty in the domain of computer vision based quality control. The expected impact of an automatized nacre thickness measurement is a steadying of the export flux of Tahitian pearls as well as an increase of customer satisfaction due to the more precise measurement. The next steps of our project comprise an automated X-ray image acquisition and an automated routing of pearls into and out of the X-tray machines. In the medium term, our project aims at extending the current $2 \mathrm{D}$ measurement to a three-dimensional one, for which the pearls have to be rotated within the X-ray machine.

\section{ACKNOWLEDGMENTS AND FUNDING}

We thank Hinano Teanotoga, Cedrik Lo and Vaihere Mooria from the DRMM for supporting our project RAPA (Reconnaissance Automatique de la Qualité des Perles de Tahiti) by providing us access to their facilities and for the supply of manually classified X-ray images. We thank as well all involved employees of the DRMM for their help and for sharing their knowledge and professional experience to support our work. The presented work is partially funded by the Ministry of Overseas France for which the authors are grateful.

\section{REFERENCES}

[1] Sturman, N.: "The Microradiographic Structures of Non-Bead Cultured Pearls," GIA Lab Notes 20th August 2009, (2009).

[2] Krzemnicki, M. S., Friess, S. D., Chalus, P., Hänni, H. A., \& Karampelas, S.: "X-ray computed microtomography: distinguishing natural pearls from beaded and non-beaded cultured pearls," Gems and Gemology, 46(2), 128, (2010).

[3] Krzemnicki, M. S., Revol, V., Hanser, C., Cartier, L., \& Hänni, H. A.: "X-ray phase contrast and X-ray scattering images of pearls," $34^{\text {th }}$ Gemological Conference Vilnius, Lithuania, (2015).

[4] Ju, M. J., Lee, S. J., Min, E. J., Kim, Y., Kim, H. Y., \& Lee, B. H.: "Evaluating and identifying pearls and their nuclei by using optical coherence tomography," Optics express, 18(13), 13468-13477, (2010).

[5] Ju, M. J., Lee, S. J., Kim, Y., Shin, J. G., Kim, H. Y., Lim, Y.,\& Lee, B. H.: "Multimodal analysis of pearls and pearl treatments by using optical coherence tomography and fluorescence spectroscopy," Optics express, 19(7), 6420-6432, (2011).

[6] Tang, Y. P., Shao-jie, X., \& Zhi-liang, Z.: "Research on Pearl Detecting and Grading Based on Monocular Multi-view Machine Vision.” British Journal of Applied Science \& Technology, 4(15), 2136, (2014).

[7] Bai, Z. L., Wen, Q. R., \& Yang, M.: “A Pearl Automatic Sorting System Based on Image Identification,” in Applied Mechanics and Materials, Vol. 496, pp. 1574-1577, (2014).

[8] Lei, M., Sun, Y., Wang, D., \& Li, P.: “Automated thickness measurements of pearl from optical coherence tomography images," in Hybrid Intelligent Systems, 2009. HIS'09. Ninth International Conference on (Vol. 1, pp. 247-251). IEEE, (2009).

[9] Sun, Y., \& Lei, M.: “Automated thickness measurements of nacre from optical coherence tomography using polar transform and probability density projection," in Intelligent Signal Processing and Communication Systems (ISPACS), 2010 International Symposium on (pp. 1-4). IEEE, (2010).

[10] Liu, J., Tian, X. L., \& Sun, Y. K.: "Pearl Thickness Measurements from Optical Coherence Tomography Images," in Applied Mechanics and Materials (Vol. 421, pp. 415-420), (2013).

[11] Loesdau, M, Chabrier, S, Gabillon, A.: "Automatic Nacre Thickness Measurement of Tahitian Pearls," in Proceedings of the 12th international conference ICIAR 2015, Canada. Lecture Notes in Computer Science, Vol. 9164, pp 446-455. Springer International Publishing, (2015). 